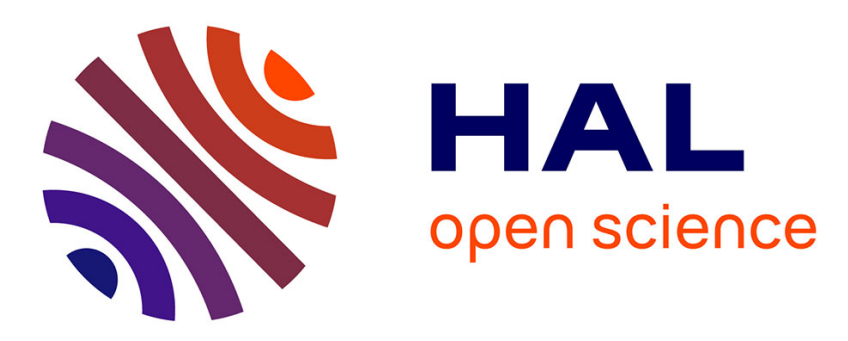

\title{
Integration and evaluation of social competences such as humor in an artificial interactive agent
}

Matthieu Riou, Bassam Jabaian, Stéphane Huet, Thierry Chaminade, Fabrice

Lefèvre

\section{- To cite this version:}

Matthieu Riou, Bassam Jabaian, Stéphane Huet, Thierry Chaminade, Fabrice Lefèvre. Integration and evaluation of social competences such as humor in an artificial interactive agent. the 1st ACM SIGCHI International Workshop, Nov 2017, Glasgow, United Kingdom. pp.41-42, 10.1145/3139491.3139495 . hal-01807782

\section{HAL Id: hal-01807782 \\ https://hal.science/hal-01807782}

Submitted on 16 Feb 2019

HAL is a multi-disciplinary open access archive for the deposit and dissemination of scientific research documents, whether they are published or not. The documents may come from teaching and research institutions in France or abroad, or from public or private research centers.
L'archive ouverte pluridisciplinaire HAL, est destinée au dépôt et à la diffusion de documents scientifiques de niveau recherche, publiés ou non, émanant des établissements d'enseignement et de recherche français ou étrangers, des laboratoires publics ou privés. 


\section{Integration and Evaluation of Social Competences such as Humor in an Artificial Interactive Agent}

\author{
Matthieu Riou \\ CERI-LIA, University of Avignon \\ Avignon, France \\ matthieu.riou@alumni.univ-avignon.
}

fr

\author{
Bassam Jabaian \\ CERI-LIA, University of Avignon \\ Avignon, France \\ bassam.jabaian@univ-avignon.fr
}

\author{
Stéphane Huet \\ CERI-LIA, University of Avignon \\ Avignon, France \\ stephane.huet@univ-avignon.fr
}

\author{
Thierry Chaminade \\ INT, University of Aix Marseille \\ Marseille, France \\ thierry.chaminade@univ-amu.fr
}

\author{
Fabrice Lefèvre \\ CERI-LIA, University of Avignon \\ Avignon, France \\ fabrice.lefevre@univ-avignon.fr
}

\begin{abstract}
In this paper, we present a brief overview of our ongoing work about artificial interactive agents and their adaptation to users. Several possibilities to introduce humorous productions in a spoken dialog system are investigated in order to enhance naturalness during social interactions between the agent and the user. We finally describe our plan on how neuroscience will help to better evaluate the proposed systems, both objectively and subjectively.
\end{abstract}

\section{CCS CONCEPTS}

- Human-centered computing $\rightarrow$ Sound-based input / output; $\bullet$ Computing methodologies $\rightarrow$ Neural networks; Adversarial learning;

\section{KEYWORDS}

artificial agent, neural network, social interactions, humorous productions

\section{ACM Reference Format:}

Matthieu Riou, Bassam Jabaian, Stéphane Huet, Thierry Chaminade, and Fabrice Lefèvre. 2017. Integration and Evaluation of Social Competences such as Humor in an Artificial Interactive Agent. In Proceedings of 1st ACM SIGCHI International Workshop on Investigating Social Interactions with Artificial Agents (ISIAA'17). ACM, New York, NY, USA, 2 pages. https: //doi.org/10.1145/3139491.3139495

\section{INTRODUCTION}

Interactive artificial agents, as spoken dialog systems, are used in many applications such as technical support services or reservation systems (for flights, accommodation, restaurant, etc.). For a while, systems used patterns and rules (e.g. [11]) to define their behaviors. Lately, those systems have been replaced or enhanced with stochastic-based approaches (e.g. [13]). Those new systems, besides having better performance, can offer more variations in

Permission to make digital or hard copies of part or all of this work for personal or classroom use is granted without fee provided that copies are not made or distributed for profit or commercial advantage and that copies bear this notice and the full citation on the first page. Copyrights for third-party components of this work must be honored.

For all other uses, contact the owner/author(s).

ISIAA'17, November 13, 2017, Glasgow, UK

(c) 2017 Copyright held by the owner/author(s)

ACM ISBN 978-1-4503-5558-2/17/11 . \$ \$15.00

https://doi.org/10.1145/3139491.3139495 their answers, avoiding unnatural repetitions. Their downside is that they require a large amount of data to be trained.

To improve user experience and engagement, some systems include social competences, such as expressions of emotions [8]. In our ongoing work, we are interested in the insertion of humorous productions in a spoken dialog system. This paper investigates the possibilities offered by stochastic approaches to generate humorous answers from an artificial agent. Section 2 reports current works on spoken dialog systems, then Section 3 investigates several ways to introduce humor in an artificial agent and Section 4 presents our ongoing work to evaluate our systems not only subjectively but also objectively. We conclude our discussions in Section 5.

\section{ONLINE ADAPTATION OF SPOKEN DIALOG SYSTEM}

This section presents ongoing work to develop a complete artificial agent, based on stochastic approaches, for goal-directed dialog. A dialog system is mainly composed of three main components: the spoken language understanding (SLU) which translates the user's verbal input into an internal representation of the information for the system, the dialog manager which controls the dialog and decides what to answer, and the natural language generation (NLG) which translates the internal representation of the system's answer into an answer in a natural language understandable by the user.

Lately, stochastic approaches made a breakthrough in natural language processing and are now used by state-of-the-art systems for the SLU (e.g. [3, 6]) and the dialog manager (e.g. [4, 5, 7]). NLG is still mainly based on template-based models, that turn out to produce good results given a specific task. Recently, stochastic approaches have also been used for NLG to address two main drawbacks of template-based approaches: lack of scalability for usage in large unconstrained domains and frequent repetition of identical and mechanical utterances.

In a previous work, we proposed a new stochastic model for NLG, based on [13], and a framework to adapt it online [12]. A first version of the model is bootstrapped with a minimal corpus and then extended by collecting new training data during actual interactions with users, using a bandit algorithm to choose whether or not the system needs to ask the user an alternative utterance. 


\section{INTEGRATION OF SOCIAL INTERACTIONS IN AN ARTIFICIAL AGENT: HUMOROUS PRODUCTIONS}

The introduction of mechanisms reproducing human sense of humor in artificial agents is a particularly interesting possibility to improve their social competence. Of course, we do not expect to reproduce the full capacity of humor that human can have, which is hard to define and describe and generally requires deep context and cultural knowledge. Our approach is rather to identify some regular mechanisms and to automatize them in order to produce a normally unexpected conversational event, endowing the agent with a more sympathetic behaviour.

Previous work in linguistic [10] established a taxonomy of humor mechanisms in social interactions. Several types of humorous productions are considered. They can be in reaction to a user's input or generated independently of it. In the first case, an opportunity is detected and reacted to. It is for example the case of humor based on polysemic terms, which the system can pretend to understand in an inadequate meaning given the context. In the second case, this is the need for facilitation in the dialog which is detected, such as a misunderstanding, and an independent humorous utterance is produced, like a pun or a joke. For this purpose, a predefined database of jokes can be used to select one according to the context.

The reactive type seems a better way to implicate the user, since it doesn't interrupt the dialog; yet it can be more difficult to produce because it requires a good understanding of the context and the meaning of words. On the other hand, the generative type can be seen as a ranking issue where we want to select the best joke within a predefined database depending on the current context.

In a second phase, we are interested in optimizing this new capacity using reinforcement learning. As users can enjoy or reject agent's humor, we want to allow the system to adapt its use of humor mechanisms, both in quantity and quality (which type of humor is appreciated, which is not). We plan to use a similar approach to the one we described in 2, and let the system choose, at each step, whether or not it is profitable to produce a humorous utterance.

\section{EVALUATION WITH HUMANS}

One of the main issues with the social competence of an artificial agent is to evaluate it. Measures like BLEU-4 [9] allow us to score a system's answer based on reference utterances, while repeatedly achieving the dialog goal (if it has one, for example booking a hotel or solving a user's technical issue) indicates a valid dialog system. However, those are only objective metrics best fit for taskoriented systems and do not measure the subjective appreciation of the system by the user. This subjective evaluation is even more interesting with a system integrating humorous productions, since it is hypothesized that these productions will improve the social interactions, therefore impacting positively the user's perception of the agent's empathy.

To measure it, we are planning to use measures of human physiology like skin conductance (peripheral nervous system) and brain imaging (central nervous system) in order to compare humanhuman and human-machine interactions during a natural conversation [1]. In particular, we could compare physiological responses to the machine depending on whether the humorous mechanisms are activated or not. Such measures can provide us with accurate information to evaluate our systems, since they are informative about actual emotional reactions of the users [2]. Furthermore, using several systems capable of humor or not, at different levels, could provide more interesting results about whether, and how, humans change their perception of the machine being interacted with and eventually adopt an intentional stance towards it [14].

\section{CONCLUSION}

In this paper, we discussed several possibilities to integrate mechanisms to produce humorous utterances in an interactive artificial agent. Two phases are foreseen. First we need to recognize and formalize regular humorous mechanisms to automate them. Then we have to optimize those mechanisms using reinforcement learning and online adaptation learning approaches. To evaluate the social competence of artificial agents endowed with humor, we can't only use objective metrics but we also need reliable subjective evaluation of the user's feelings. To do this evaluation, we intend to draw on social cognitive neuroscience approaches. Thus, we aim to bring together neuroscience and artificial agents, to be mutually profitable, in order to both improve those artificial agents and increase our knowledge about human social interactions.

\section{ACKNOWLEDGEMENTS}

This work has been partially carried out within the Labex BLRI (ANR-11-LABX-0036).

\section{REFERENCES}

[1] Thierry Chaminade, Léo Biaocchi, Farah H Wolfe, Noël Nguyen, and Laurent Prévot. 2015. Communicative Behavior and Physiology in Social Interactions. In Proceedings of the 1st Workshop on Modeling INTERPERsonal SynchrONy And infLuence. ACM, 25-30.

[2] T. Chaminade, M. Zecca, S. J. Blakemore, A. Takanishi, C. D. Frith, S. Micera, P. Dario, G. Rizzolatti, V. Gallese, and M. A. Umilta. 2010. Brain response to a humanoid robot in areas implicated in the perception of human emotional gestures. PLoS One 5, 7 (2010), e11577.

[3] Emmanuel Ferreira, Bassam Jabaian, and Fabrice Lefèvre. 2015. Zero-shot semantic parser for spoken language understanding. In INTERSPEECH.

[4] Emmanuel Ferreira and Fabrice Lefèvre. 2015. Reinforcement-learning based dialogue system for human-robot interactions with socially-inspired rewards. Computer Speech \& Language 34, 1 (2015), 256-274.

[5] Emmanuel Ferreira, Grégoire Milliez, Fabrice Lefèvre, and Rachid Alami. 2015. Users' Belief Awareness in Reinforcement Learning-based Situated Human-Robot Dialogue Management. In IWSDS.

[6] Emmanuel Ferreira, Alexandre Reiffers-Masson, Bassam Jabaian, and Fabrice Lefèvre. 2016. Adversarial Bandit for Online Interactive Active Learning of Zero-shot Spoken Language Understanding. In ICASSP.

[7] Grégoire Milliez, Emmanuel Ferreira, Michelangelo Fiore, Rachid Alami, and Fabrice Lefèvre. 2014. Simulating human-robot interactions for dialogue strategy learning. In SIMPAR.

[8] Magalie Ochs, Radosław Niewiadomski, Catherine Pelachaud, and David Sadek. 2005. Intelligent expressions of emotions. Affective computing and intelligent interaction (2005), 707-714.

[9] Kishore Papineni, Salim Roukos, Todd Ward, and Wei-Jing Zhu. 2002. BLEU: a method for automatic evaluation of machine translation. In ACL.

[10] Béatrice Priego-Valverde. 1999. L'humour dans les intéractions conversationnelles: jeux et enjeux. Ph.D. Dissertation. Aix-Marseille 1.

[11] Owen Rambow, Srinivas Bangalore, and Marilyn Walker. 2001. Natural Language Generation in Dialog Systems. In HLT.

[12] Matthieu Riou, Bassam Jabaian, Stéphane Huet, and Fabrice Lefèvre. 2017. Online adaptation of an attention-based neural network for natural language generation. In INTERSPEECH.

[13] Tsung-Hsien Wen, Milica Gašić, Dongho Kim, Nikola Mrkšić, Pei-Hao Su, David Vandyke, and Steve Young. 2015. Stochastic Language Generation in Dialogue using Recurrent Neural Networks with Convolutional Sentence Reranking. In SIGDIAL.

[14] Agnieszka Wykowska, Thierry Chaminade, and Gordon Cheng. 2016. Embodied artificial agents for understanding human social cognition. Phil. Trans. R. Soc. B 371, 1693 (2016), 20150375. 